\title{
La investigación sobre identidad y ciudadanía en Estados Unidos: de la nueva historia social a la nueva historia cultural
}

\author{
Bárbara Weinstein \\ Department of History State University of \\ New York at Stony Brook
}

\begin{abstract}
Resumen
Este artículo analiza las nuevas tendencias en la historiografía norteamericana sobre ciudadanía e identidad. La autora argumenta que el cambio de la nueva historia social a la nueva historia cultural también significa una transición del estudio de grupos marginalizados u oprimidos (mujeres, americanos africanos, gays) al estudio de posiciones de subjetividad y relacionales (género, raza, queerness) que son mucho más radicales en sus implicaciones. Afirma que la nueva historia cultural surgió de tendencias culturalistas en la historia social, pero el cambio permite repensar sustancialmente ciertas cuestiones. La nueva historia cultural no sólo amplia el espacio para la discusión de asuntos como género, raza y sexualidad, transfiriendo estos temas de la periferia al centro del análisis histórico, sino también permite empezar a desconstruir las categorías liberales clásicas que han conformado las discusiones sobre ciudadanía.
\end{abstract}

\begin{abstract}
This article deals with the new tendencies in North American historiography about citizenship and identity. It argues that the move from new social to new cultural history has also meant a shift from the study of marginal or oppressed groups (women, African-Americans, gays) to the study of subject positions and relations (gender, race, "queerness") that are much more radical in their implications. It contends that the new cultural history grew out of culturalist tendencies in social history, but also allows for substantial rethinking of certain questions. Not only does the new cultural history broaden the terrain for the discussion of issues such as gender, race and sexuality, moving such themes from the periphery to the center of historical analysis, but allowing us to begin to deconstruct the classic liberal categories that have informed discussions of citizenship.
\end{abstract}


Para entender las nuevas tendencias de la historiografía norteamericana respecto a la identidad y ciudadanía, es necesario regresar a la época anterior, al auge de la "nueva historia social" en los años 70 y comienzos de los 80 . A pesar de la impresión que circula actualmente entre los historiadores acerca de la gran ruptura entre la historia social de aquella época, con su orientación neomarxista, y la nueva historia cultural, con el "sesgo lingüístico", y las visiones posmodemas de hoy, creo más adecuado afirmar que las tendencias actuales representan desdoblamientos de las preocupaciones anteriores ${ }^{1}$. Aún hoy, las nuevas tendencias historiográficas. inclusive sobre identidad y ciudadanía, se inspiran en una crítica de los conceptos fundamentales de la (ya vieja) nueva historia social.

La cuestión de ciudadanía, lejos de ser una preocupación para la nueva historia social en los Estados Unidos, fue una categoría, en cierta forma despreciada, por lo menos en su sentido tradicional (el ciudadano del concepto liberal, el sujeto autónomo, relacionado como individuo con el Estado). Entre los historiadores de la época (incluso yo), la noción de ciudadanía fue criticada desde dos perspectivas Primero, se consideró como una identidad que reforzó la noción de "consenso" en la historia norteamericana y que privilegió la identidad individual, para esconder el significado y la importancia de la clase en la historia de los Estados Unidos. Segundo, la noción de ciudadanía fue utilizada tradicionalmente, por los historiadores de la posguerra, sin sentido crítico y sin atención a los enormes grupos de habitantes de Estados Unidos que no gozaban de los plenos derechos de ciudadanía afroamericanos, mujeres, inmigrantes no blancos, homosexuales- ${ }^{2}$.

Por eso, la gran misión de la historiografía de la nueva historia social fue la recuperación de la categoría de clase y un énfasis en la actuación/resistencia de los grupos

\footnotetext{
${ }^{1}$ Los siguientes artículos discuten el impacto del "giro lingüístico" y otras tendencias posmodemas: Frank Ankersmit, "Historiography and Postmodernism", History and Theory 28 (1989): 137-53; John Toews. "Intellectual History After the Linguistic Turn: The Autonomy of Meaning and the Irreducibility of Experience", American Historical Review (en adelante citado como AHR) 92 (octubre 1987): 879-907; Alan Megill, "Recounting the Past: 'Description', Explanation, and Narrative in Historiography", AHR 94 (Junic 1989): 627-53; J. Walkowitz, M. Jehlen y B. Chevigny, "Patrolling the Borders: Feminist Historiography and the New Historicism", Radical History Review (en adelante citado como RHR) 43 (invierno 1989): 23-43.

${ }^{2}$ Sobre los presupuestos masculinos y etnocéntricos del lenguaje "universal" de los historiadores y antropólogos de la posguerra, véase David A. Hollinger, "How Wide the Circle of the 'We'? American Intellectual and the Problem of the Ethnos since World War II," AHR 98 (abril 1993): 317-37.
} 
oprimidos mediante los procesos de asimilación y represión. Fue una reacción contra la narrativa de la época progresista que representaba los programas educacionales y asistenciales de aquel período, como una gran fuerza por la transformación (de la masa de inmigrantes o migrantes de diversos orígenes) para una población de ciudadanos relativamente homogénea, de buen comportamiento y respetable ${ }^{3}$. En vez de esta viñon de asimilación (a la de identidad "americana" o a la de clase media) y de la eficiencia de la clase dirigente, hubo, un fuerte énfasis en las resistencias de las clases subalternas, y en la manutención de los espacios autónomos de los grupos populares ${ }^{4}$.

Indirectamente, la bibliografía de esta orientación contribuyó a complicar y cuestionar la noción "fundacionalista" de ciudadano. Pero esta no fue una preocupación explícita de aquella historiografía. Aun cuando la ciudadanía entró en discusión, por ejemplo, en estudios del discurso republicano de los trabajadores (como el de Sean Wilentz) o en estudios del "americanismo" de la clase laboral (como el de Gary Gerstle), el objetivo del historiador fue más el de destacar el sentido de clase en estos discursos ${ }^{5}$. La identidad de clase fue entendida como "real", mientras la ciudadanía fue todavía un concepto que disfrazó otras identidades potencialmente más perturbantes del orden social ${ }^{6}$.

\footnotetext{
${ }^{3}$ Oscar Handlin, The Americans: A New History of the People of the United States (Boston: Little, Brown, 1963) es un buen ejemplo de la historiografía que destacó la "asimilación" de los grupos étnicos en el cuerpo politico.

${ }^{4}$ Entre las obras más importantes de la nueva historia social, ver Herbert Gutman, Work, Culture, and Society in Industrializing America (New York: Random House, 1977); David Montgomery, Workers' Control in America: Studies in the History of Work, Technology, and Labor Struggles (Cambridge: Cambridge University Press, 1979) y Alan Dawley, Class and Community: The Industrial Revolution in Lynn (Cambridge: Harvard Universtiy Press, 1976). Aún durante su auge, la nueva historia social fue criticada por historiadores como Eugene Genovese y Tony Judt, por la tendencia a exagerar la autonomía de las clases populares y a ignorar la esfera política.

${ }^{5}$ Sean Wilentz, Chants Democratic: New York City and the Rise of the American Working Class, 1788-1850 New York: Oxford University Press, 1984); Gary Gerstle, Working Class Americanism: The Politics of Labor in Textile City, 1914-1960 (Cambridge: Cambridge University Press, 1989). ${ }^{6}$ Para un ejemplo de eso, véase Bárbara J. Fields, "Ideology and Race in American History", en Region, Race, and Reconstruction, ed. J. Morgan Kousser y James M. McPherson (New York: Oxford University Press, 1982), 143-77. Fields hace un contraste entre la raza y la clase, insistiendo que la raza es una construcción puramente ideológica, en cuanto clase tiene una base material y objetiva. Un reciente libro sobre historiografía escrito por tres eminentes historiadoras norteamericanas delinea la transición de la historia social a la historia cultural, pero su análisis tiende a confundir las distinciones entre la visión materialista/sociocultural asociada con E. P. Thompson y la nueva historia cultural posmoderna: Joyce Appleby, Lynn Hunt y Margaret Jacobs, Telling the Truth about History (New York: W. W. Norton, 1995), 220-21.
} 
En cuanto a los estudios de la mujer, del negro, del indio en la nueva historia social, estas investigaciones se presentaron inicialmente al margen de la profesión. Paulatinamente, estas "categorías" se impusieron cada vez más, pero la tendencia fue todavía en al sentido de "adicionarlas". Entre los historiadores de esta línea llegó a ser oportuno hablar un poco de raza o género, de la recreación y del consumo, pero las figura centrales de la historiografía seguían siendo hombres blancos de la clase trabajadora en el centro de producción. ${ }^{7}$

En los últimos quince años, con el crecimiento de la política de identidades en Estados Unidos, y el desarrollo de programas para los estudios de la mujer, de los afroamericanos, y de los nuevos grupos étnicos, los historiadores comenzaron a revisar la narrativa modelo de la nueva historia social. La raza, la etnicidad y el género (y recientemente, la orientación sexual) se convirtieron en categorías cada vez más significativas, reconocidas como "identidades" que no pueden ser reducidas a una "conciencia" o "cultura" de clase. Estrechamente relacionada con esta tendencia fue el cambio del lugar de investigación de la fábrica u otro lugar de trabajo o producción, hacia la casa, la familia, la calle, el barrio, los centros de recreación, la redes de amistad y hasta el mundo de las emociones. Actividades como el consumo llegaron a ser objeto de estudio muy significativos, y no sólo categorías secundarias, subordinadas a las actividades "productivas"8.

Otro cambio muy importante en la profesión del historiador norteamericano (y probablemente similar a la situación que ocurre actualmente en el Brasil) fue la migración de muchos jóvenes y algunos viejos investigadores, aquellos que anteriormente hacían historia social, al terreno de la "nueva historia cultural". Además de esa reorientación del objeto de investigación (la literatura, la música, la cultura popular, etc.), lo que más distingue a la nueva historia cultural (de la nueva historia social) es su énfasis en los modos de "representación" y en la construcción cultural/ discursiva de identidades, subjetividades, etc., que reflejan un influenciado "sesgo

\footnotetext{
${ }^{7}$ Para una discusión de los límites del método de "incluir a la mujer en el retrato", véase Joan Wallach Scott, "Women's History", en Gender and the Polines of History (New York: Columbia University Press, 1988), 15-27.

${ }^{8}$ Iris Berger, Elsa Barkley Brown y Nancy A. Hewitt, "Symposium: Women, Blacks and Workers Confront Gender, Race and Class," Feminist Studies 18 (verano 1992): 283-326.
} 
lingüístico". Cuando el historiador social hablaba de trabajo, él se refería a la propia "experiencia" de los operarios en el proceso de trabajo; cuando la historiadora cultural habla de trabajo, ella quiere decir la construcción de diferentes nociones de trabajo, y del lenguaje usado para hablar de eso (el discurso sobre el trabajo, y no la experiencia del trabajo) ${ }^{9}$.

En este sentido, la nueva historia cultural abrió un espacio para recuperar el problema de ciudadanía. Si todas las identidades, comunidades, etc., son "imaginadas" o "inventadas" o "socialmente construidas", entonces el concepto de ciudadano no es ni más ni menos "real", y llega a ser un tema legítimo e importante para estudiar. Hablar de ciudadanía no sería necesariamente aceptar la "realidad" de la categoría, o aceptar una definición "liberal" de ciudadanía.

Para elaborar estas nuevas tendencias, voy a referirme primero a algunos debates entre los historiadores sociales y los historiadores culturales que indican ciertas rupturas significativas de la vieja con la nueva orientación ${ }^{10}$. Después, trataré detalladamente algunos trabajos recientes que indican nuevas direcciones de investigación, pero también indican nítidas continuidades con la historiografía de los años 70.

Algunos historiadores sociales, como es de esperar, se han sentido amenazados por las nuevas tendencias. Hay una línea crítica contra el énfasis en el texto y en el lenguaje, y contra el aparente rechazo de una realidad o verdad histórica estable y comprensible $^{11}$. Para dar un buen ejemplo, en 1996 la revista Radical History Review circuló, en internet, una convocatoria para artículos destinados a un número dedicado al problema de la pobreza, esto es, la construcción de la pobreza como un problema social, una categoría política, etc. Un conocido historiador social, Roger Horowitz, respondió muy decepcionado que la propia RHR estaba hablando de pobreza como una construcción social; dice él: "los pobres entienden muy bien

\footnotetext{
${ }^{9}$ Appleby, Hunt y Jacobs, Telling the Truth about History, 217-23.

${ }^{10}$ Ibíd. En cuanto a Appleby, Hunt y Jacobs usan la categoría "historia social" para denominar la historia demográfica y cuantitativa, la "vieja" historia social, me estoy refiriendo en este artículo a la nueva historia social, con sus influencias más nítidamente neomarxistas.

${ }^{11}$ Un buen ejemplo de eso es Bryan Palmer, Descent into Discourse: The Reification of Language and the Writing of Social History (Philadelphia, 1990).
} 
lo que es pobreza"12. Al final, él criticó a la revista por haber abandonado su compromiso con las luchas de los pobres y los explotados. Es un ejemplo transparente de la idea que la experiencia determina el conocimiento y la conciencia sin ninguna medición de cualquier otra influencia cultural, o ningún problema más complejo de subjetividad.

Pero, a pesar de episodios de esta clase, la disciplina histórica no padeció el tipo de "crisis" que sufrieron las áreas de literatura y antropología en los Estados Unidos durante los últimos años. Creo interesante el preguntarse por qué la nueva orientación lingüística, el posmodernismo, etc., no provocaron una crisis más profunda en la profesión. A primera vista, estas nuevas tendencias presentaban un desafío bastante serio a los modos convencionales de hacer historia. Ellas ponían en duda toda empresa de reconstrucción del pasado. Pero la reacción ha sido relativamente moderada. Hay una serie de debates en la profesión, pero el tono de discusión es extremadamente discreto comparado con las "guerras" en las otras disciplinas. Creo que la explicación es la existencia tanto de fuertes continuidades como de discontinuidades entre la nueva historia social y la nueva historia cultural. La nueva historia social ya predicaba la necesidad de estudiar grupos antiguamente considerados marginales, e intentaban entender la formación de conciencia a través de la esfera cultural. ${ }^{13}$ Su crítica implícita de las viejas nociones de ciudadanía, y de las afirmaciones unlversalizantes de la vieja historia progresivista, creó una base para repensar el concepto de ciudadanía. Esto no quiere decir que no hay nada de nuevo en la nueva historia cultural, pero el impacto no fue tan significativo en la profesión de historia como sí en otras disciplinas.

El resultado es una sucesión de debates serios, pero en cierta forma amistosos. Un punto importante de contestación es la cuestión de la resistencia o "agency"14. Por ejemplo, Linda Gordon, una de las más conceptuadas historiadoras de su generación

\footnotetext{
${ }^{12}$ Roger Horowitz, Reply to RHR Cali for Papers on Internet.

${ }^{13}$ Por eso, una figura como Raymond Williams puede funcionar como una referencia extremadamente importante, tanto para la historia social como para la historia cultural. Appleby, Hunt y Jacobs, Telling the Truth about History, 221.

${ }^{14}$ La palabra "agency" se refiere a la capacidad de los seres humanos de influir en el curso de la historia o de ser "agentes" de la historia, y generalmente es utilizada en contraste con el estructuralismo, que, supuestamente, minimiza el papel activo de los seres humanos.
} 
en las áreas de historia social y femenina, escribió un libro sobre mujeres de la clase trabajadora y violencia doméstica que se llama Heroes of Their Own Lives. Ella siguió en el libro una línea que es nítidamente un producto de la nueva historia social (con su propio "sesgo feminista"). Según Gordon, muchas mujeres en esa situación, amenazadas, al mismo tiempo, por maridos abusivos y por asistentes sociales que querían retirar a los niños de la "casa problemática", resistieron a esas invasiones en su familia y rehusaron a ser víctimas, bien de sus maridos, bien de los profesionales de la clase media. La idea central es que esas mujeres usaban los valores que venían de la experiencia de las mujeres de la clase trabajadora para resistir los valores e imposiciones de la clase dominante. Rechazaron la tentativa de los representantes del Estado y de la clase media para imponer sus propias nociones de buena ciudadana o de buena madre. Joan Scott, otra historiadora muy conocida, feminista, y una discípula convicta del sesgo lingüístico, criticó el libro de Gordon a pesar de su noción central de "agency". Scott, en contraste, enfatiza en la formación de subjetividades, y la participación de esas mujeres en el mismo campo cultural, con los mismos conceptos y discurso de la "buena madre", que funcionan como la fundación de sus reivindicaciones de ciudadanía. Scott critica a Gordon por naturalizar la resistencia de esas mujeres, y por marginalizar la reacción menos combativa de las otras que, implícitamente, no tiene "agency" en la óptica de Gordon. Scott, entretanto, acota que la misma "pasividad" es una estrategia discursiva para cultivar el apoyo de la asistente social, y así modificar las relaciones de poder, sin que se pueda calificar a un grupo como "héroes con agency" y a otro grupo como "víctimas pasivas". ${ }^{15}$

Otro campo de debate es la historia de la clase trabajadora, un lugar privilegiado para la nueva historia social, y hoy un terreno bastante contestado y criticado. En este contexto, Joan Scott aparece otra vez, ahora para criticar a E. P. Thompson y su énfasis en la "experiencia", que tenía gran impacto en la historiografía de la nueva historia social, con su atención a los trabajadores "comunes", en vez de las autoridades o (todavía peor) de las vanguardias. Scott reconoce la experiencia como un concepto fundacional de la obra thompsoniana; así, la "experiencia" existe para Thompson como una categoría no problemática. El significado de la experiencia

\footnotetext{
${ }^{15}$ Linda Gordon, Heroes of Their Own Lives: The Politics and History of Family Violence (New York: Viking, 1988); respecto al debate, véase Joan Scott y Linda Gordon, "Review and Reply", Signs 15 (verano 1990): 848-60.
} 
laboral es previsible para Thompson y es para él el punto de origen de la conciencia, en cuanto para Scott el significado de la experiencia depende enteramente del contexto cultural y de la subjetividad ya construida y continuamente reconstruida. Aún más, ella muestra cómo Thompson coloca ciertas experiencias laborales (como la popularidad de la figura mesiánica de Joanna Southcott) fuera de la narrativa modelo de la clase trabajadora inglesa, caracterizándolas como femeninas. El resultado es, dice ella, la construcción de una identidad laboral "normativa" y "masculinizada"16.

En muchos estudios recientes, se nota todavía una tensión entre el énfasis anterior en la experiencia, agency, y resistencia, frente a las preocupaciones posmodernas. Pero, en general, los estudios más importantes e influyentes de los últimos años siguen una línea de la nueva historia cultural, con su énfasis en la construcción del campo cultural a través de los discursos y de las prácticas sociales, y estos estudios generalmente destacan los límites de la resistencia, o hasta rechazan el concepto de resistencia como un proceso que ocurre fuera del campo cultural dominante. Uno de los resultados de esta tendencia es cuestionar aún más la narrativa "heroica" creada por los historiadores de la década del 70 (tanto sobre trabajadores como mujeres, grupos étnicos u homosexuales). Claro que los propios historiadores sociales ya habían complicado esa narrativa, destacando el racismo, el machismo y la homofobia de los trabajadores blancos, e indicando las divisiones entre mujeres por raza o por clase. Sería exagerado decir que aquella generación no tuvo conciencia de eso. Pero las categorías fueron siempre trabajadas, en primer lugar, como cosas separadas (así, el racismo podía ser expulsado del movimiento laboral si los trabajadores reconocían su verdadera identidad de clase, con la implicación de que el racismo funcionaba independientemente de la esfera de posición social) ${ }^{17}$. Había una tendencia de estudiar la raza o el género o la orientación sexual apenas en relación con los grupos nítidamente marginalizados o explotados en función de

\footnotetext{
${ }^{16}$ Scott, "Women in The Making of the English Working Class," en Gender and the Politics of History, 6890; para una lectura feminista de Thompson menos crítica, véase Catherine Hall, "The Tale of Samuel anc Jemima: Gender and Working Class Culture in Early Nineteenth Century England," en White, Male anc Middle Class (New York: Routledge, 1992), 124-50.

${ }^{17}$ Para una crítica desde esta perspectiva, véase David R. Roediger, The Wages of Whiteness: Race and tht Making of the American Working Class (Londres: Verso, 1991), 6-11.
} 
las categorías en cuestión. Entonces, el problema de la raza sólo apareció en el contexto de las experiencias de los trabajadores negros; la cuestión de género sólo apareció en los estudios de las mujeres. Tal vez la gran contribución de ciertos trabajos recientes es justamente repensar y criticar esas maneras de abordar el tema.

Después de un período en el que la "raza" quedaba subordinada a la "clase" en las conversaciones históricas (véase los trabajos de Eugene Genovese o Barbara Fields), la identidad racial o étnica se han vuelto una preocupación absolutamente central de los historiadores norteamericanos. Y sobre todo, las ópticas de los historiadores en relación con ese tema ya cambiaron bastante.

En el pasado, hablar de "raza" quería decir hablar de "negro" o afroamericano, raras veces era hablar de los "indios" o de los chinos; pero generalmente la raza era una cuestión relacionada con los negros o con otras poblaciones "marginalizadas" o discriminadas. El resto de la población, en cierta forma, no tenía "raza", o, mejor dicho, su identidad racial no era una cuestión para interrogar o un problema histórico. La identidad racial de los grupos oprimidos fue considerada, generalmente, como una cosa fija, dada, y no el resultado de un largo y complicado proceso histórico ${ }^{18}$.

En contraste, en los últimos años hubo una fuerte insistencia en el concepto de identidad racial como construcción, y una construcción "relacionar" (es decir, nuestra noción de negro depende de nuestra noción de blanco). Un resultado, el concepto de "blancura", whiteness, y los discursos y prácticas (y los privilegios y "sacrificios") asociados con ese estatus. En otras palabras, la "raza" hace parte de todas las construcciones sociales; y estará, de alguna forma, siempre presente. Y aún más: muchos historiadores argumentan que es imposible "separar" nociones de clase o género de nociones de raza ${ }^{19}$. La historiadora Nancy Hewitt explica esta relación usando el lenguaje de la química: las identidades no son "mezclas" en las

\footnotetext{
${ }^{18}$ Hubo siempre importantes afirmaciones, como Edmund S. Morgan, American Slavery, American Freedom: The Ordeal of Colonial Virginia (New York: 1975) que fue uno de los primeros libros que trató la esclavitud, no como una institución "peculiar", un desvío del camino de la libertad, sino como un aspecto intrínseco a la libertad de los blancos.

${ }^{19}$ Berger, Barkley y Hewitt, "Symposium". Véase también Ava Baron, "Gender and Labor History", en Work Engendered: Toward a New History of American Labor, ed. Baron (Ithaca: Cornell University Press, 1991).
} 


\section{FRONTERAS}

cuales cada elemento mantiene su integridad; identidad se asimila a la "reacción química" que vincula y transforma todos los elementos ${ }^{20}$.

Quiero citar dos estudios importantes que trabajan con el concepto de blancura. Un libro pionero es el de David Roediger; Wages of Whiteness investiga la formación de la clase trabajadora en el siglo pasado y la creciente asociación de ciertas cualidades del buen trabajador y ciudadano (disciplinado, sobrio, buen padre de familia) con la "blancura" ${ }^{21}$. Roediger elabora el proceso de la formación de una identidad restringida a los trabajadores blancos (una frase, en este contexto, repetitiva: ser trabajador era ser hombre y blanco), con énfasis en la disciplina y en un cierto tipo de comportamiento en la esfera pública que traía ciertos privilegios. Pero Roediger destaca también los sacrificios de la blancura; según él, para estos hombres blancos, había un persistente deseo o nostalgia de un orden no sujeto a la disciplina industrial, y en la vida cultural de los blancos ese mundo "no reglamentado" fue asociado cada vez más a los negros.

Es así como Roediger trata de entender el enorme suceso, en la cultura popular blanca entre la Guerra Civil y la Primera Guerra Mundial, del "blackface minstrelsy"22 . Antiguamente visto simplemente como un medio de ridiculizar a los negros, o una expresión (aunque racista) de resentimientos populares, esa forma cultural y teatral tiene un doble sentido para Roediger, que percibe un tipo de "nostalgia" en la música y en la actuación de estos artistas. O sea, gozando de los "privilegios" de la blancura, el blanco de las clases populares ahora sólo puede quedar fuera de control cuando él se disfraza de "negro" (o mejor, de su proyección

\footnotetext{
${ }^{20}$ Nancy A. Hewitt, "Symposium: Compounding Differences", 318.

${ }^{21}$ Otros libros que trabajan con el concepto de "blancura" incluyen Roediger, Towards the Abolition of Whiteness: Essays on Race, Politics, and Working-Class History (Londres: Verso, 1994); Alexander Saxton, The Rise and Fall ofthe White Republic (Londres, 1990); Noel Ignatiev, How the Irish Became White (New York: Routledge, 1995); Ann Laura Stoler, Race and the Education of Desire (Durham: Duke University Press, 1995).

22 "Blackface Minstrelsy" se refiere a un tipo de teatro musical y popular en el cual los músicos se pintaban los rostros (con tinta negra) y cantaban el "dialecto de los negros". Los cantantes eran todos hombres (pero muchos se disfrazaban de mujer), y la gran mayoría blancos, pero hubo algunos grupos teatrales de negros que adoptaron el estilo de blackface minstrelsy. Además del estudio de Roediger, "White Skins, Black Masks: Minstrelsy and White Working Class Formation in the Antebellum United States," en The Wages of Whiteness, 115-31, véase Eric Lott, Love a Theft: Blackface Minstrelsy and the American Working Class (New York: Oxford University Press, 1993).
} 
de la identidad negra). El placer de una cierta sensualidad, de ciertas formas de recreación y de varias maneras de expresión son prohibidas al blanco, que necesita adoptar temporalmente una identidad caricaturizada y "negra" para recuperar este placer.

Otro historiador que tiene ciertas preocupaciones comunes a las de Roediger, es Matthew Jacobson, cuyos libros Special Sorrows y Whiteness of a Dijferent Color, investigan la relación entre la inmigración, el imperialismo y la definición inestable de "whiteness" en la sociedad norteamericana ${ }^{23}$. El primero de estos dos libros examina la trayectoria política y cultural de las tres comunidades de inmigrantes en los Estados Unidos, judíos, irlandeses y polacos, en el fin del siglo XIX y en los inicios del siglo XX. Jacobson escoge estos tres grupos porque cada uno de ellos se consideraba un pueblo sin estado, un pueblo "exiliado", que tenía una cierta simpatía con los movimientos de los subditos coloniales fuera de Europa. Jacobson comienza su historia en la época de la lucha de Cuba contra España y la entrada de los Estados Unidos en la guerra que desembocó en la reducción de Puerto Rico, las Filipinas y, en cierta forma, de Cuba al dominio colonial de los Estados Unidos. Usando los periódicos, novelas, canciones y poesía que circulaban en esas comunidades étnicas, Jacobson muestra que en el inicio de esta época los portavoces culturales de los tres grupos étnicos se identificaron claramente con las luchas de los pueblos no libres contra los poderes coloniales, y se manifestaron nítidamente contra la posibilidad de que Estados Unidos se volviera un imperio colonial ${ }^{24}$.

Pero el discurso público de esa época ponía a Estados Unidos cada vez más como un poder civilizador, y a los pueblos de esas sociedades como salvajes o bárbaros. Así, los grupos étnicos ahora estarían apoyando, no a pueblos luchado por la libertad, en el mismo sentido que los irlandeses, judíos y polacos luchaban por su propia emancipación, sino a personas de color oscuro representadas como bárbaras ${ }^{25}$.

\footnotetext{
${ }^{23}$ Matthew Frye Jacobson, Special Sorrows: The Diasporic Imagination of Irish, Polish, and Jewish ímmigrants in the United States (Cambridge: Harvard University Press, 1995); Whiteness of a Dijferent Color: European ímmigrants and the Alchemy of Race (Cambridge: Harvard University Press, 1998).

${ }^{24}$ ídem, Special Sorrows, 141-76. Esto es en el sentido del imperialismo formal; en el sentido informal, Estados Unidos ya había entrado en el concurso imperialista.

${ }^{25}$ Stuart Creighton Miller, "Benevolent Assimilation": The American Conquest of the Philipines, 1899-1903 (New Heaven: Yale University Press, 1982); Amy Kaplan, "Black and Blue on San Juan Hill", en Cultures of United States Imperialism, ed. Amy Kaplan y Donald E. Pease (Durham: Duke University Press, 1993).
} 


\section{FRONTERAS}

Jacobson traza la paulatina transformación de la cultura política de esas comunidades, que se asociaban (con sus preocupaciones políticas) cada vez menos con los pueblos no-europeos. Una vez más, el punto de división era la "blancura", estos tres grupos étnicos (que antiguamente fueron considerados como razas distintas) comenzaron a percibirse como integrantes de la raza blanca y ciudadanos de un imperio poderoso, en vez de ser los pueblos refugiados identificados con las luchas de las personas de color en otras partes del mundo.

El creciente énfasis, en la historia del género, en la masculinidad (o "manliness"), está relacionado con esta nueva tendencia de estudiar la "blancura". Muchos historiadores del género, del mismo modo que los estudiosos de la raza, resolvieron reconocer que no son apenas las mujeres las que tienen género, y comenzaron a adoptar una perspectiva relacional. Intimamente ligada con la literatura sobre la "blancura", los trabajos sobre masculinidad investigan los cambios en las "normas" de comportamiento para los hombres, y el papel de raza y de clase en la construcción de esas normas. Por ejemplo, en términos de sexualidad, el hombre "ideal" de la clase media se distingue por su capacidad de dominar sus instintos sexuales, una capacidad para autocontrol que es altamente masculina. En contraste, la supuesta incapacidad de los hombres de las clases bajas (o de las razas oscuras) de controlarse sexualmente, lejos de ser una señal de su hipermasculinidad, es una señal de su estado infantil o su debilidad; aún más, un hombre tan descontrolado es capaz de buscar satisfacción en una relación homosexual, que sería la mejor prueba de que él no es lo suficientemente masculino. (Eso indica, también, la marcada distinción entre el "machismo" y el concepto norteamericano de "manliness"). Claro que este mismo discurso, mutatis mutandi, fue aplicado por los defensores de la cultura burguesa para demostrar la debilidad y corrupción de la vieja aristocracia ${ }^{26}$.

\footnotetext{
${ }^{26}$ Estudios históricos sobre masculinidad incluyen Gail Bederman, Manliness and Civilization: A Cultural History of Gender and Race in the United States, 1880-1917 (Chicago: University of Chicago Press, 1995): E. Anthony Rotundo, American Manhood: Transformations in Masculinity from the Revolution to the Modern Era (New York: Basic Books, 1993); J. A. Mangan y James Walvin, eds., Manliness and Morality: Middle-Class Masculinity in Britain and America, 1800-1940 (New York: St. Martin's, 1987); Mrinalini Shina, Colonial Masculinity: The Manly Englishman and the Effeminate Bengali in the Late Nineteenth Century (Manchester: Manchester University Press, 1995); una discusión pionera de la masculinidad en México es Steve J. Stern, The Secret History of Gender: Women, Men, and Power in Late Colonial Mexico (Chapel Hill: University of North Carolina Press, 1995), cap. 7.
} 
Al mismo tiempo, los nuevos trabajos sobre género muestran que los discursos sobre la masculinidad cambiaron de una época a otra. En la mitad del siglo pasado hubo un énfasis constante en el autocontrol, en el trabajo, en la independencia y en la represión de los instintos primitivos (hasta decir que los hombres anglosajones habían superado esas inclinaciones). En las últimas décadas del siglo, en contraste, hubo una preocupación en la excesiva "civilización" (inclusive "feminización") del hombre de la clase media. Él continuaba siendo obligado a controlarse, a trabajar, ser buen padre de familia, pero también se deseó que mantuviera una cierta "cultura física", participara en actividades atléticas y experimentara la naturaleza recreativamente en la caza, la pesca y el camping. El hombre ideal se convirtió en un tipo como Teddy Roosevelt, el hombre "civilizado" que además, enfrenta los desafíos del mundo primitivo y sabe canalizar los instintos primigenios. Él era el hombre ideal para la época imperial.

Gail Bederman, en un reciente libro, Manliness and Civilization, analiza el discurso de civilización y muestra cómo este fue compuesto a partir de ciertos discursos de "raza" y de "género", incluyendo, la noción común a finales del siglo pasado, que en la alta civilización hay una extrema diferenciación entre los papeles del hombre y de la mujer ${ }^{27}$. Entre los muchos ejemplos de estos discursos elaborados en el libro, ella incluyó la exposición colombiana en Chicago en 1893, una feria conmemorativa del cuarto centenario del "descubrimiento" del Nuevo Mundo. El sector más importante de esta exposición era la "White City ", literalmente, la ciudad blanca, refiriéndose al color de las edificaciones, pero que tenía un doble significado que era imposible de ignorar. Fue en la "White City" donde los organizadores colocaron los símbolos del progreso, la industria, la modernidad, todo explícitamente atribuido a la creatividad y disciplina del hombre (y del hombre implícitamente blanco). Hubo varios intentos de grupos feministas o negros por ganar "representación" en la ciudad blanca; las mujeres ganaron un pequeño edificio en la periferia de la ciudad, para exhibir las contribuciones de la mujer a la civilización, pero los negros fueron negados de cualquier representación en este sector de la exposición. (Los "africanos",

\footnotetext{
${ }^{27}$ Bederman, Manliness and Civilization, 35-36. Este concepto nos ofrece un fuerte contraste con las ansiedades de los años 20 del siglo XX, cuando la modernidad "amenazaba" apagar las distinciones entre hombre y mujer. Véase Susan K. Besse, Restructuring Patriarchy: The Modernization of Gender Inequality in Brazil, 1914-1940 (Chapel Hill: University of North Carolina Press, 1996), cap. 1.
} 
por eso, fueron "representados" en el sector que se llamó "Midway Plaisance ", donde hubo recreaciones de varias culturas agrarias, comenzando con una aldea de la campiña alemana, y "bajando" hasta una aldea "africana" del Dahomey). Bederman, además de indicar la marginalización o exclusión de estos grupos, a pesar de sus esfuerzos por ser incluidos, muestra que las mujeres y los negros también actuaron en el terreno del discurso sobre civilización. Las feministas acabaron excluyendo a las mujeres negras de su comisión, y su pequeña exhibición reprodujo la asociación de la modernidad y del progreso con la blancura. Los negros (representados por dos figuras de renombre, Frederick Douglass e Ida B. Wells) enfatizaron el papel del hombre negro, o su "manliness", e insistieron en que los hombres negros en los Estados Unidos eran hasta más "civilizados" que los hombres blancos (quienes participaban en barbaridades como los linchamientos). Aún más: ellos se disociaron de la "aldea africana" de tal manera que mostraba su crítica no sólo como una cuestión de la naturaleza de la exhibición. Esto significa que también en estos "contradiscursos" hubo una fuerte tendencia a aceptar ciertos presupuestos de los conceptos de blancura y de masculinidad como base de civilización (y por implicación, como base de la ciudadanía en el mundo civilizado) ${ }^{28}$.

En cuanto a la raza y al género, se convirtieron en las categorías más importantes en la literatura reciente, hay un creciente interés también en la categoría de orientación o preferencia sexual (que, obviamente, tiene muchas conexiones con la literatura y los debates sobre masculinidad). Respecto a la orientación sexual, hay dos tendencias marcadas. Una es un tipo de historia social del homosexualismo, parecida a los estudios hechos en la década pasada para recuperar la historia de la mujer $^{29}$. Pero los mejores de los recientes estudios, como Gay New York,

\footnotetext{
${ }^{28}$ Bederman, de otro lado, insiste que este contradiscurso de civilización abrió un espacio para enfrentar y repensar el discurso hegemónico. Douglass, por ejemplo, escribió que el negro estaba "resistiendo como hombre" su represión racista, y colocó el racismo fuera de la cultura de los civilizados. Bederman, Manliness and Civilization, 39.

${ }^{29}$ Algunos estudios centrales son John D'Emilio y Estelle Freedman, Intímate Matters: A History of Sexuality in America (New York: Harper and Row, 1988); Martin Duberman, Martha Vicinus, y George Chaunceyl eds., Hidden from History: Reclaiming the Gay and the Lesbian Past (New York: NAL, 1989); Elizabeth Kennedy y Madeline Davis, Boots of Leather, Slippers of Gold: The History of a Lesbian Community (New York: Routledge, 1993); Lilian Faderman, Odd Girls and Twilight Lovers: A History of Lesbian Life inTwentieth Century America (New York: Columbia University Press, 1991); Alian Berubé, Corning Out Under Fire: The History of Gay Men and Women during World War Two (New York: Free, 1990).
} 
de George Chauncey, no se contentan con la simple recuperación de un pasado homosexual $^{30}$. Chauncey, en su recreación vivida de la subcultura "gay" en New York antes de Stonewall (1969), insiste en que nuestra noción de que en aquella época los hombres gays se quedaban en el armario, saliendo y exhibiéndose apenas en lugares muy escondidos y marginales, se basa en una imagen retorcida y excesivamente simplificada de la época. En contraste, él muestra que hubo una cultura homosexual vibrante en New York antes de Stonewall, y que muchos hombres transitaron entre los mundos gay y no-gay sin grandes problemas ${ }^{31}$. Aún más: él argumenta que la militancia de hoy y la afirmación de la identidad homosexual como una identidad "totalizante" o esencial, acaban dificultando esta relación fluida entre los mundos gay y no-gay; así, actualmente no existe la misma flexibilidad, y en cierta forma los homosexuales viven en un mundo más segregado que antiguamente.

Este argumento de Chauncey encaja muy bien con el proyecto de los discípulos de la queer theory ${ }^{32}$. No viendo el homosexualismo como una identidad fija (y aún menos biológica), los historiadores influenciados por la queer theory no buscan recuperar la historia de los homosexuales como un grupo marginalizado, o reconstruir la lucha heroica de los militantes gays de la época de Stonewall. En cambio, se interesan más en la historia de la inestabilidad del deseo sexual y de la identidad sexual. Semejante a la historia de la mujer y la historia del género, los historiadores de la queer theory quieren mostrar que no es necesario tener la presencia de un sujeto homosexual para hacer la historia de los queerness, para leer un subtexto de ambigüedad sexual, o para desdoblar las ansiedades culturales y políticas sobre la

\footnotetext{
${ }^{30}$ George Chauncey, Gay New York: Gender, Urban, Culture, and the Making of the Gay Male World, 18901940 (New York: Basic, 1994).

${ }^{31}$ La Rebelión de Stonewall (1969), un importante acto de resistencia en Greenwich Village contra los policías, sirve para muchos escritores como división entre la vieja y la nueva cultura gay. Sobre este episodio, véase Martin Duberman, Stonewall (New York: Dutton, 1993).

${ }^{32}$ Queer es, en el lenguaje convencional, una palabra mal educada para gays, semejante a "marica" en español. Fue escogida por los adeptos de la teoría, exactamente por causa de ser mal educada e indiscreta. Además de eso, queer puede connotar una persona que es "anormal", pero ella no identifica la sexualidad del sujeto de manera tan definitiva como la palabra "gay". En las palabra de Martha Umphrey, "queer history puede abarcar las prácticas heterosexuales no normativas", junto con la historia gay. "The Trouble with Harry Thaw", RHR 62 (primavera 1995): 23. Ver también Donna Penn, "Queer: Theorizing Politics and History", RHR 62 (primavera 1995): 24-42; Michael Warner, ed., Fear of a Queer Planet: Queer Politics and Social Theory (Minneapolis: University of Minnesota Press, 1993).
} 
"amenaza" del homosexualismo o los peligros de la sexualidad descontrolada. Como un historiador explicó en un reciente artículo, los adeptos de la queer theory se interesan más en el significado histórico de la música de Cole Porter, que influía sobre la cultura en general, que en la recuperación de figuras poco conocidas de la comunidad gay $^{33}$. Del mismo modo que la historia del género y la historia de la raza están cambiando de un enfoque en los grupos marginales u olvidados, para hacer énfasis en la centralidad de las cuestiones de raza y género, los historiadores de orientación sexual están adoptando una visión que rechaza el esencialismo, y privilegia la construcción de identidad a través de discursos y prácticas. Especialmente, los tres están insistiendo en poner sus preocupaciones en el centro del proceso histórico y recalcan que esas cuestiones son de importancia para todos los historiadores. Entonces, la "clase" no será una categoría que debe preocupar apenas a los que estudian a los trabajadores; el "género" no es una categoría que debe preocupar apenas a los que estudian a las mujeres; la "raza" no es una categoría que debe preocupar apenas a los que estudian a los afroamericanos; la "orientación sexual" no es una categoría que debe preocupar apenas a los que estudian a los homosexuales. Integrar estas categorías en un proyecto histórico no es simplemente una cuestión de hablar de una mujer u otra, o incluir una figura gay o negra. Todos los discursos y prácticas, insisten estos recientes trabajos, se construyen y son superpuestos con los discursos de género, raza, etc.

Finalmente, ¿cuáles son las implicaciones de estas nuevas tendencias historiográficas respecto a la cuestión de la ciudadanía? Como lo mencioné en la introducción, es difícil entender los desdoblamientos de la nueva historia cultural en esta cuestión sin considerar las críticas de la "ciudadanía" que surgieron de la (vieja) nueva historia social. La perspectiva crítica de la nueva historia social garantizó que la historia de los grupos excluidos no acabara rindiendo una narrativa "inspiracional" (y conservadora en sus implicaciones) de un proceso, a veces arduo y conflictivo, pero inevitable a la expansión de la categoría de ciudadano, para incluir al trabajador, a la mujer, al negro, al homosexual, en cada grupo, y así demostrar su capacidad de ejemplificar los valores y los comportamientos del buen ciudadano

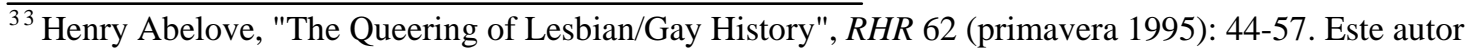
destaca la fuerte influencia de Michel Foucault en los historiadores de esta nueva generación.
} 
norteamericano. Los historiadores de los años 70, con su fuerte rechazo a la noción consensual de la política, iniciaron (tal vez inconscientemente) la deconstrucción del concepto de ciudadanía.

Por eso, su énfasis en los grupos marginalizados, el uso de las categorías de clase, género o raza como "dadas" y no problemáticas, y el papel central de la "experiencia" (también no problematizada) generaron una corriente esencialista que rechazó cualquier noción de asimilación ${ }^{34}$. Esta tendencia se reforzó en un momento histórico que testimonió el crecimiento de varios movimientos sociales basados en la política de identidad. En cambio de predicar la igualdad o la integración (antiguamente el tema de los movimientos sociales), el discurso esencialista destacaba y festejaba la diferencia, ahora dándole a esta diferencia un sentido positivo. Así, por ejemplo, la cultura femenina era diferente y superior a la cultura masculina ${ }^{35}$. Los afroamericanos se interesaron menos en participar en la política del "mainstream", e insistieron en el derecho de conservar su diferencia cultural y criar una cierta autonomía política $^{36}$. Aún más: los movimientos sociales generalmente intentaban basar sus reivindicaciones en una historia de la opresión que dependía de una imagen del grupo como una entidad fija y relativamente homogénea, y con claras señales para distinguir quién pertenecía y quién no pertenecía al grupo.

Los problemas creados por esas corrientes esencialistas no son apenas intelectuales. Este tipo de esencialismo depende, en el último instante, de la marginalidad; él dificulta las alianzas entre diferentes movimientos sociales, y genera muchos conflictos internos. Al final, ¿quién va a determinar cuál es la propia esencia de la

\footnotetext{
${ }^{34}$ Un ejemplo de la tendencia antiasimilacionista es Daniel Harris, The Rise and Fall of Gay Culture (New York: Hyperion, 1997); véase también la crítica de George Chauncey de este libro en The New York Times Book Review, domingo 7 de septiembre 1997, 20.

${ }^{35}$ Un texto clave para esta posición es Carol Guilligan, In a Different Voice: Psychological Theory and Women's Development (Cambridge: Harvard University Press, 1992).

${ }^{36}$ Este movimiento "separatista" o nacionalista es generalmente asociado con la escuela "afrocéntrica" cuyo objetivo es desvendar la contribución cultural, científica y filosófica de África a la civilización mundial, y en los casos extremos, demostrar superioridad esencial de los africanos. Molefi Kete Asante, Kemet, Afrocentricity and Knowledge (Trenton: Africa World, 1990). Para una crítica del Afrocentricity, véase Paul Gilroy, The Black Atlantic: Modernity and Double Consciousness (Cambridge: Harvard University Press, 1993), 187-96.
} 
identidad femenina, o negra, o gay? Luego quedó claro que la tensión entre "igualdad y diferencia" llevó a ciertas implicaciones imprevistas. El mejor ejemplo de eso es el caso "Sears". Un grupo de mujeres que trabajaban en Sears procesaron a la empresa por haber excluido a las mujeres de los cargos más lucrativos de los almacenes. La Sears se defendió usando el libro de una historiadora feminista (que públicamente se colocó a favor de la mujeres que procesaban a Sears), mostrando que la misma historiadora, Alice Kessler-Harris, reconoció en su propio libro que las mujeres tradicionalmente cambiaron el salario por horarios más flexibles o por condiciones de trabajo más relajadas, porque sus prioridades eran diferentes de las de los hombres. Ella insistió en el libro que la trabajadora era diferente del trabajador (no sólo en el sentido de "construcción" sino también en el sentido "real"), y usaba sus propios instrumentos de resistencia y negociación. ¿Cuál fue el resultado de este discurso de diferencia? La Sears ganó ${ }^{37}$.

Entonces, de cierta forma, los trabajos de la nueva historia cultural, y los nuevos conceptos de identidad, pueden ser vistos como un intento de encontrar una salida de la siguiente dicotomía: o usted se asimila al "mainstream", a los valores normativos de la ciudadanía norteamericana, o usted rechaza la asimilación y privilegia su estado "marginal", su alteridad.

La preocupación actual es crear una tercera opción que rechace las identidades y categorías fijas y transhistóricas, mas no apenas en relación con los grupos "marginales". Por el contrario, el mayor interés es mostrar cómo las construcciones de blancura y masculinidad formaron la base de ciudadanía, y por eso, aún con los nuevos discursos o las nuevas construcciones, sin una deconstrucción de estas "categorías modelo", vamos a testimoniar la continua exclusión, desvalorización y subordinación de grandes carnadas de la población. La blancura, según David Roediger, es una categoría completamente vacía en el sentido positivo; en sus propias palabras: "No es que la blancura sea falsa y opresiva; es que la blancura es apenas falsa y opresiva y nada más". En su argumento la blancura no tiene otro contenido. En contraste, él insiste en que la negritud (Blackness) no es una esencia,

\footnotetext{
${ }^{37}$ Alice Kessler-Harris, Out to Work: A History of Wage-Earning Women in the United States (New York: Oxford University Press, 1982); Scott, "The Sears Case," en Gender and the Politics of History, 167-77; Ruth Milkman, "Women's History and the Sears Case", Feminist Studies 12 (1986): 394-95.
} 
pero al mismo tiempo reconoce que esta identidad tiene una historia con implicaciones positivas para la comunidad afroamericana ${ }^{38}$. De la misma manera, los nuevos trabajos sobre masculinidad cuestionan la reciente tendencia a festejar la identidad masculina, además supuestamente desde una perspectiva no machista ${ }^{39}$. Pero si la masculinidad es un concepto construido con la propuesta de valorizar a los hombres en relación con las mujeres, cualquier intento de alabar o reafirmar la masculinidad implica el fortalecimiento de una identidad hegemónica.

En un sentido más amplio, esta visión rechaza la noción de categorías normativas y monta una crítica más vigorosa y completa de la noción de "mainstream ". Así, el proceso de crear un concepto realmente justo y equitativo de ciudadanía no es una cuestión de incluir los negros junto con los blancos o las mujeres junto con los hombres o los gays junto con los heterosexuales. Pero sí es una cuestión de abolir las categorías políticas del blanco, del hombre y del heterosexual. El gran problema en la cuestión de la identidad y la ciudadanía no es el falso esencialismo de los grupos marginales, es el falso y opresivo esencialismo de los grupos dominantes. Finalmente, la nueva historiografía de los años 90 ha introducido la noción de ciudadanía, pero con la clara intención de cuestionar y deconstruir todos los elementos que constituyen este concepto.

\footnotetext{
${ }^{38}$ Roediger, introducción a Abolition of Whiteness.

${ }^{39}$ Catherine Stimpson, prólogo a Manliness and Civilization, por Bederman.
} 